\title{
Characterization of Robust Solution Quadratically Constrained Quadratic Optimization Problem Subjected to Data Uncertainty
}

\author{
Moussa BARRO ${ }^{1}$, Satafa SANOGO², Mohamed ZONGO ${ }^{3}$ and Sado TRAORÉ ${ }^{4}$
}

\begin{abstract}
Robust Optimization (RO) arises in two stages of optimization, first level for maximizing over the uncertain data and second level for minimizing over the feasible set. It is the most suitable mathematical optimization procedure to solve real-life problem models. In the present work, we characterize robust solutions for both homogeneous and non-homogeneous quadratically constrained quadratic optimization problem where constraint function and cost function are uncertain. Moreover, we discuss about optimistic dual and strong robust duality of the considered uncertain quadratic optimization problem. Finally, we complete this work with an example to illustrate our solution method.
\end{abstract}

Mathematics Subject Classification: (2010) 90C20 - 90C26 - 90C46-90C47

Keywords: Robust Optimization, Data Uncertainty, Quadratic Optimization Strong Duality, Robust Solution, DPJ-Convex.

1 Department of Mathematics, University Nazi BONI.

2 Polytechnic school of Ouagadougou.

3 Department of Mathematics, University Nazi BONI.

4 Department of Mathematics, University Nazi BONI.

Article Info: Received: March 10, 2021. Revised: April 9, 2021.

Published online: April 28, 2021. 


\section{Introduction}

The present paper is devoted to the characterization of the solutions of optimization problems which are affected by data uncertainty. This means that all the data of the problem are neither known nor available when its optimal solution has to be computed. Such an optimization model assumes the usual one but it is clearly different. In fact, it allows to handle model error, to take into account imperfections, additive noises or external uncertainties. For examples: (a) An entrepreneur must estimate the time required to finish a job. Uncertainties due to the labor market, the supply of materials, bad weather conditions, etc. constitute unknown parameters to consider; (b) In robot design, even if the movement model is carefully planned to provide a stable locomotion, a humanoid robot is very likely to fall due to the uncertainties induced by the irregularities in the ground; (c) When designing a nuclear power plant, engineers have to consider the uncertainties about earthquakes, the strength of beams, etc. Moreover, one cannot always ignore uncertainties during modeling process (i.e. always considering a determinist model) because that could lead to security issues in real-world applications (e.g., designing nuclear power installations, autonomous car control system, aeronautical structures optimization). Then, it is certain that optimization model subject to data uncertainty is most adequate methodology for solving real-word problems: it is Robust Optimization (RO). In a nutshell, RO provides a solution which is immunized against the effect of parameters uncertainty: that solution is called robust solution $[1,2,5]$. Nevertheless, there is a similarity between RO and Stochastic Optimization (SO). The latter optimization model can be considered like a particular case of RO where the uncertainty set distribution is assumed to follow a probability law. Note that stochastic optimization is wholly a very important field of research and profound to be developed here. However, for more details, we recommend the works of Peter Kall and Stein W. Wallace [32], John R. Birge and François Louveaux [9], Alexander Shapiro, Andrzej Ruszczyński et al. [46,47], Kurt Marti [37], George B. Dantzig and Gerd Infanger $[15,27]$.

Concerning the concept of robustness and uncertainty in mathematical programming models, it was shyly started with the pioneer works of S. K. Gupta, J. Rosenhead [22,44], G. B. Dantzig [15], F. J. Gould, and A. L. Soyster who first called it Inexact Linear Programming" [21,49]. Afterwards, the topic of RO was quickly expanded through mainly the contributions of J. M. Mulvey et al. [38], P. Kouvelis, and Gang Yu [34], L. El Ghaoui et al. [17,18], A. Ben-Tal and A. Nemirovski [2,3,4,5], D. Bertsimas and M. Sim [6,7,8,48], V. Jeyakumar et al. [30,31,35]. In these last years, RO has been a focus within many research communities starting with the field of control, convex optimization, mathematical programming, or even economics, and many fields of engineering science [2,26,33]. Basically, whenever an optimization problem is formulated, the question arises whether really all parameters and inputs are exactly known and what changes if they are not. In this sense, it is not surprising that many researchers were and are attracted by the challenges of robust optimization.

Finally in this work, our purpose is to analyze Quadratically Constrained Quadratic Optimization Problems (QCQOPs) subjected to uncertain data both in the constraint function and cost function. And we next investigate the characterization of their solutions. In fact, QCQOPs are very important in their own right but not enough studies with uncertainties. This choice is also motivated by the fact that QCPOPs are in some ways the general forms of almost all mathematical programming models. In addition, the literature about analysis of quadratic forms [13,16,19, 42], quadratic programming and QCQOP solution methods (basically indeterminist form) is well-supplied with well-known and sophisticated techniques.

For instance, they occur as subproblems in methods for numerical/algorithmic optimization 
problems such as Newton-Lagrange methods (Sequential Quadratic Programming (SQP), InteriorPoint Method (IMP), BFGS (Broyden, Fletcher,Goldfarb, and Shanno) method) in one hand. In other hand, any smooth optimization problem can be approximated with a quadratic model (thought of doing second order Taylor series approximation). Even if the considered problem is not enough smooth, it could be approximated quadratically. For this technique, we refer to the topic of Derivative Free Optimization (DFO) methods, see for example the introductory book of Andrew R. Conn, Katya Scheinberg and Luis N. Vicente [14]. We also recall that under some basic assumptions (at least symmetry and semi-dene positivity) on the data of a QCQOP, we can get convex or Second Order Conic Optimization Problem (SOCP), see Section 2. It is well-known that in the field of optimization an ideal and wanted framework is the convexity one $[5,10,11,20,23,24,25,28,29,31,36,39,40,43,45]$ since that can make easiest to solve exactly optimization problems (i.e., the characterization of their global solution).

With the uncertainties, this advantage is in the most of time difficult to have in RO without wellthought-out assumptions.

The rest of the paper is organized as follows. Section 2 recall some preliminaries and describes the Quadratically Constrained Quadratic Optimization Problem Model which will be study throughout this paper. In Section 3, we give some fundamental results basically joint-range convexity conditions and S-Lemma.

The Section 4 is dedicated to the characterization of robust optimal solutions for an uncertain quadratic optimization problem with respect to homogeneous case and non-homogeneous one. In Section 5, we compare optimistic dual and robust strong dual of the uncertain optimization problem. We deal with an example in the homogeneous case in the Section 6 for an illustration. Section 7 concludes our work.

\section{Quadratically Constrained Quadratic Optimization Problem Model}

In this section, we start by recalling some basic definitions and fixing some notations. In what follow, $\mathbb{R}$ denotes the set of real numbers, $\mathbb{R}^{n}$ denotes the linear space of $n$-dimension vector and $\mathbb{S}_{n}(\mathbb{R})$ denotes the space of $\mathrm{n} \times \mathrm{n}$ symmetric matrices with real entries. For all, $\mathrm{A}, \mathrm{B} \in \mathbb{S}_{n}(\mathbb{R})$, $A \geqslant 0$ (respectively $A>0$ ) means that the matrix $A$ is positive semi-definite (respectively $A$ is positive definite). By analogy, $A \geqslant B$ (respectively $A>B$ ) is equivalent to $A-B \geqslant 0$ (respectively $\mathrm{A}-\mathrm{B}>0$ ). For any twice differentiable function $\mathrm{q}: \mathbb{R}^{n} \rightarrow \mathbb{R}, \nabla q$ denotes the gradient (first derivative) of $\mathrm{q}$ and $\nabla^{2} \mathrm{q}$ denotes the Hessian matrix (second derivative) of q. Given a set $\mathrm{E}$, intE denotes the interior of E. If $E_{1}$ and $E_{2}$ are subsets of a set E, then the set $E_{1}+E_{2}=\left\{e=e_{1}+e_{2} \in E \mid\left(e_{1}, e_{2}\right) \in E_{1} \times E_{2}\right\}$. The segment $\left[v_{1}, v_{2}\right]$ is defined by $\left[v_{1}, v_{2}\right]=\left\{v \mid v:=t v_{2}+(1-t) v_{1}, t \in[0,1]\right\}$. A set $\mathrm{C}$ is convex if the line segment between any two points in $\mathrm{C}$ lies in $\mathrm{C}$ i.e., if for any $c_{1}, c_{2} \in C$ and any $\lambda \in[0,1]$, we have $\lambda c_{1}+$ $(1-\lambda) c_{2} \in C$. A function $\mathrm{f}: \mathbb{R}^{n} \rightarrow \mathbb{R}$ is convex if and only if its epigraph is a convex set.

A general formulation of a quadratic optimization problem under uncertainty can be formulated as follows:

$$
\begin{aligned}
& \min _{x \in \mathbb{R}^{n}} \frac{1}{2} x^{T} A_{u} \mathrm{x}+a_{\omega}^{T} \mathrm{x} \quad(\mathrm{QCQP}) \\
& \text { s.t. } \frac{1}{2} x^{T} B_{v} \mathrm{x}+b_{\varsigma}^{T} \mathrm{x}+\beta \leq 0
\end{aligned}
$$

where: $\beta \in \mathbb{R}, a_{\omega}, b_{\varsigma} \in \mathbb{R}^{n}, A_{u}, B_{v} \in \mathbb{S}_{n}(\mathbb{R})$, and $(\mathrm{u}, \mathrm{v}, \omega, \varsigma)$ belongs to the uncertainty set $\mathcal{U}$. In addition, we use an affine interpolation to define uncertainty data. Then, we set : 
$A_{u}=A_{0}+u A_{1}, B_{v}=B_{0}+v B_{1}, a_{\omega}=a_{0}+\omega a_{1}, b_{\varsigma}=b_{0}+\varsigma b_{1}$,

with

$A_{0}, A_{1}, B_{0}, B_{1} \in \mathbb{S}_{n}(\mathbb{R})$ and $a_{0}, a_{1}, b_{0}, b_{1} \in \mathbb{R}^{n}, \mathcal{U}$ is an Euclidian product of sets and given by $\mathcal{U}=\left[u_{1}, u_{2}\right] \times\left[v_{1}, v_{2}\right] \times\left[\omega_{1}, \omega_{2}\right] \times\left[\varsigma_{1}, \varsigma_{2}\right]$.

We also put ;

$u_{1}=\left[u_{1}, u_{2}\right], u_{2}=\left[v_{1}, v_{2}\right], u_{3}=\left[\omega_{1}, \omega_{2}\right]$ and $u_{4}=\left[\varsigma_{1}, \varsigma_{2}\right]$.

\section{Convex Analysis of Quadratic forms and S-procedures}

The convex analysis and the study of quadratic forms are the essential ingredients to concoct a good recipe in the field of optimization. Indeed, in a convex world or in the presence of quadratic forms, the characterization of the solutions for an optimization problem can be carried out without major difficulty. This is why, R. T. Rockafellar stated that the great watershed in optimization is not between linearity and nonlinearity, but convexity and non-convexity (Rockafellar 1993, [11]). In this work, we use mainly joint-range convexity results for quadratic forms which were initially proposed by L. L. Dines [16] and improved later by other authors such that B.T. Polyak [42]. In addition, these convexity properties of quadratic are helpful to prove some results like Lemma 1, Theorem 2 and Corolary 1: we talk about S-procedure for generality. The S-Lemma is a useful tool especially in Control Theory and RO.

Theorem 1 (L. L. Dines (1941) [16])

Given, $\mathrm{A}, \mathrm{B} \in \mathbb{S}_{n}(\mathbb{R})$. Then the set $\left\{\left(x^{T} A x, x^{T} B x\right) \mid x \in \mathbb{R}^{n}\right\}$ is convex.

The generalization of convexity results for more than two homogeneous quadratic forms was established by B.T. Polyak [42] and given as follows:

Proposition 1 ([42])

Let $A_{1}, \ldots, A_{m} \in \mathbb{S}_{n}(\mathbb{R})$. If the matrices $A_{1}, \ldots, A_{m}$ commute, then the set

is closed convex cone. $\left\{\left(x^{T} A_{1} x, \ldots, x^{T} A_{m} x\right) \mid x \in \mathbb{R}^{n}\right\}$

The following lemma is the homogeneous version of the famous S-lemma.

Lemma 1 (S-lemma [29,41])

Let $A_{1}, A_{2} \in \mathbb{S}_{n}(\mathbb{R})$. Assume that there exists $x_{0} \in \mathbb{R}^{n}$ such that $x_{0}^{T} A_{1} x<0$. Then, the following statements are equivalent:

(i) $\forall x \in \mathbb{R}^{n}, x_{0}^{T} A_{2} x<0 \Rightarrow x_{0}^{T} A_{1} x<0$

(ii) $\exists . \lambda \geq 0 \mid A_{1}+\lambda A_{2} \geqslant 0$.

Theorem 2 Let $A_{0}, A_{1}, B_{0}, B_{1} \in \mathbb{S}_{n}(\mathbb{R}) ; u_{1}, u_{2}, v_{1}, v_{2} \in \mathbb{R}$ such that $u_{1} \leq u_{2}$ and $v_{1} \leq v_{2}$. Let $u_{1}=\left[u_{1}, u_{2}\right], \mathcal{U}_{2}=\left[v_{1}, v_{2}\right]$, and assume that

$$
\Omega=\left\{\left(x^{T} A_{u_{1}} x, x^{T} A_{u_{2}} x, x^{T} B_{v_{1}} x, x^{T} B_{v_{2}} x\right) \mid x \in \mathbb{R}^{n}\right\} \text { is convex. }
$$


Then, for some $\alpha, \beta \in \mathbb{R}$, exactly one the following two statements holds:

(i) $\exists x \in \mathbb{R}^{n} \mid \frac{1}{2} x^{T} A_{u} \mathrm{x}<\alpha \Rightarrow \frac{1}{2} x^{T} B_{v} \mathrm{x}<0, \quad \forall(u, v) \in \mathcal{U}_{1} \times \mathcal{U}_{2}$.

(ii) $\exists\left(\lambda_{1}, \lambda_{2}\right) \in \mathbb{R}_{+}^{2} \backslash\{(0,0)\}, \exists(\bar{u}, \bar{v}) \in \mathcal{U}_{1} \times \mathcal{U}_{2}$ such that:

$\lambda_{1}\left(\frac{1}{2} x^{T} A_{\bar{u}} \mathrm{x}-\alpha\right)+\lambda_{2}\left(\frac{1}{2} x^{T} B_{\bar{v}} \mathrm{x}+\beta\right) \geq 0, \forall x \in \mathbb{R}^{n}$.

Proof $[(i i) \Longrightarrow \neg(i)]$. Assume that (ii) holds. If (i) is satisfied, then

$\exists x \in \mathbb{R}^{n} \mid \frac{1}{2} x^{T} A_{u} \mathrm{x}<\alpha$ and $\frac{1}{2} x^{T} B_{v} \mathrm{x}+\beta<0, \forall(u, v) \in \mathcal{U}_{1} \times \mathcal{U}_{2}$.

This implies that for all $\left(\lambda_{1}, \lambda_{2}\right) \in \mathbb{R}_{+}^{2} \backslash\{(0,0)\}$, we have :

$\exists x \in \mathbb{R}^{n} \mid \lambda_{1}\left(\frac{1}{2} x^{T} A_{u} \mathrm{x}-\alpha\right)+\left(\frac{1}{2} x^{T} B_{v} \mathrm{x}+\beta\right)<0, \forall(u, v) \in \mathcal{U}_{1} \times \mathcal{U}_{2}$.

Which contradicts (ii).

Let us show that $[\neg(i) \Longrightarrow(i i)]$.

Firstly, let us show that the set

$$
\boldsymbol{\Gamma}=\left\{\left(\max _{\boldsymbol{u} \in \mathcal{U}_{1}} x^{T} A_{u} \mathrm{x}, \max _{\boldsymbol{v} \in \mathcal{U}_{2}} x^{T} B_{v} \mathrm{x}\right) \mid x \in \mathbb{R}^{n}\right\}+\operatorname{int}^{2} \mathbb{R}_{+}^{2}
$$

is convex.

Let $\left(q_{1}, r_{1}\right),\left(q_{2}, r_{2}\right) \in \Gamma$ and $\delta \in[0,1]$. So, there exist $x_{1}, x_{2} \in \mathbb{R}^{n}$ such that:

$$
\left\{\begin{array}{l}
\max _{\boldsymbol{u} \in \mathcal{U}_{1}} x_{1}^{T} A_{u} x_{1}^{T}<q_{1} \\
\max _{\boldsymbol{u} \in \mathcal{U}_{1}} x_{1}^{T} B_{v} x_{1}^{T}<r_{1} \\
\max _{\boldsymbol{u} \in \mathcal{U}_{1}} x_{2}^{T} A_{u} x_{2}^{T}<q_{2} \\
\max _{\boldsymbol{u} \in \mathcal{U}_{1}} x_{2}^{T} B_{v} x_{2}^{T}<r_{2}
\end{array}\right.
$$

For all $x \in \mathbb{R}^{n}, u \mapsto \frac{1}{2} x^{T} A_{u} \mathrm{x}$ is an affine mapping with respect to (w.r.t) u. Consequently, it attains its maximum value on the compact set $\mathcal{U}_{1}$ more precisely, have:

$$
\max _{\boldsymbol{u} \in \mathcal{U}_{1}} x^{T} A_{u} \mathrm{x}=\max \left\{x^{T} A_{u_{1}} x, x^{T} A_{u_{2}} x\right\}
$$

Reasoning in same manner that previously, one has:

$$
\max _{\boldsymbol{u} \in \mathcal{U}_{2}} x^{T} B_{v} \mathrm{x}=\boldsymbol{m a x}\left\{x^{T} B_{v_{1}} x, x^{T} B_{v_{2}} x\right\}
$$


By reporting the maximum value equalities in inequality system (3), one finds:

$$
\left[\begin{array}{l}
x^{T} A_{u_{1}} x<q_{1} \\
x^{T} A_{u_{2}} x<q_{1} \\
x^{T} B_{v_{1}} x<r_{1} \\
x^{T} B_{v_{2}} x<r_{1} \\
y^{T} A_{u_{1}} y<q_{2} \\
y^{T} A_{u_{2}} y<q_{2} \\
y^{T} B_{v_{1}} y<r_{2} \\
y^{T} B_{v_{2}} y<r_{2}
\end{array}\right.
$$

It follows from the system (4) above that:

$$
\begin{aligned}
& \left(q_{1}, q_{1}, r_{1}, r_{1}\right) \in\left\{\left(x^{T} A_{u_{1}} x, x^{T} A_{u_{2}} x, x^{T} B_{v_{1}} x, x^{T} B_{v_{2}} x\right)\right\}+\text { int } \mathbb{R}_{+}^{4}, \\
& \left(q_{2}, q_{2}, r_{2}, r_{2}\right) \in\left\{\left(y^{T} A_{u_{1}} y, y^{T} A_{u_{2}} y, y^{T} B_{v_{1}} y, y^{T} B_{v_{2}} y\right)\right\}+\text { int } \mathbb{R}_{+}^{4},
\end{aligned}
$$

which is equivalent to

$$
\left\{\begin{array}{c}
\left(q_{1}, q_{1}, r_{1}, r_{1}\right) \in \Omega+\text { int } \mathbb{R}_{+}^{4} \\
\left(q_{2}, q_{2}, r_{2}, r_{2}\right) \in \Omega+\text { int } \mathbb{R}_{+}^{4}
\end{array}\right.
$$

Since $\Omega$ is convex, the set $\Omega+\operatorname{int} \mathbb{R}_{+}^{4}$ is also convex. Then $\left(\delta q_{1}+(1-\delta) q_{2}, \delta q_{1}+(1-\delta) q_{2}, \delta r_{1}+(1-\delta) r_{2}, \delta r_{1}+(1-\delta) r_{2}\right) \in \Omega+$ int $\mathbb{R}_{+}^{4}$.

Thus, there exists $z \in \mathbb{R}^{n}$ such that

Hence,

$$
\left\{\begin{array}{c}
z^{T} A_{u_{1} z}<\delta q_{1}+(1-\delta) q_{2}, \\
z^{T} A_{u_{2}} z<\delta q_{1}+(1-\delta) q_{2}, \\
z^{T} B_{v_{1}} z<\delta r_{1}+(1-\delta) r_{2} \\
z^{T} B_{v_{2}} z<\delta r_{1}+(1-\delta) r_{2} .
\end{array}\right.
$$

$$
\left\{\begin{array}{l}
\max _{\boldsymbol{u} \in \mathcal{U}_{1}} z^{T} A_{u} \mathrm{z}<\delta q_{1}+(1-\delta) q_{2} \\
\max _{\boldsymbol{u} \in \mathcal{U}_{1}} z^{T} B_{v} \mathrm{z}<\delta r_{1}+(1-\delta) r_{2} .
\end{array}\right.
$$

So, $\delta\left(q_{1}, r_{1}\right)+(1-\delta)\left(q_{2}, r_{2}\right) \in \boldsymbol{\Gamma}$ and consequently $\boldsymbol{\Gamma}$ is convex.

Secondly since (i) fails, we can separate $\boldsymbol{\Gamma}$ and the set $\{(2 \alpha,-2 \alpha)\}$. Then, by hyperplane separation theorem (also known as the geometric form of Hahn-Banach Theorem, see H. Brezis (1983) [12]), there exists $\left(\lambda_{1}, \lambda_{2}\right) \in \mathbb{R}^{2} \backslash\{(0,0)\}$ such that

$$
\begin{gathered}
\lambda_{1}\left(\max _{\boldsymbol{u} \in \mathcal{U}_{1}} \frac{1}{2} x^{T} A_{u} \mathrm{x}+\eta\right)+\lambda_{2}\left(\max _{\boldsymbol{v} \in \mathcal{U}_{2}} \frac{1}{2} x^{T} B_{v} \mathrm{x}+\varepsilon\right) \geq \\
\alpha \lambda_{1}-\beta \lambda_{2}, \forall x \in \mathbb{R}^{n}, \forall \eta, \varepsilon \in \operatorname{int} \mathbb{R}_{+} .
\end{gathered}
$$

-Suppose that $\lambda_{1}<0$. 
When $(\eta, \varepsilon)$ tends to $(+\infty, 0)$ in inequality (5), we get at the limit: $-\infty \geq \alpha \lambda_{1}-\beta \lambda_{2}$. Thus, $\lambda_{1} \geq 0$.

-In the same way, we show that $\lambda_{1} \geq 0$.

-And afterwards, one has $\left(\lambda_{1}, \lambda_{2}\right) \in \mathbb{R}_{+}^{2} \backslash\{(0,0)\}$.

We have just shown that there exists $\left(\lambda_{1}, \lambda_{2}\right) \in \mathbb{R}_{+}^{2} \backslash\{(0,0)\}$ such that:

$\lambda_{1} \max \left\{\frac{1}{2} x^{T} A_{u_{1}} x, \frac{1}{2} x^{T} A_{u_{2}} x\right\}+\lambda_{2} \max \left\{\frac{1}{2} x^{T} B_{v_{1}} x, \frac{1}{2} x^{T} B_{v_{2}} x\right\} \geq \alpha \lambda_{1}-\beta \lambda_{2}, \forall x \in \mathbb{R}^{n}$.

This implies that the following inequalities system (6) below has no solution,

Let

$$
\left[\begin{array}{l}
\frac{1}{2} x^{T}\left(\lambda_{1} A_{u_{1}}+\lambda_{2} B_{v_{1}}\right) \mathrm{x}<\alpha \lambda_{1}-\beta \lambda_{2}, \\
\frac{1}{2} x^{T}\left(\lambda_{1} A_{u_{1}}+\lambda_{2} B_{v_{2}}\right) \mathrm{x}<\alpha \lambda_{1}-\beta \lambda_{2}, \\
\frac{1}{2} x^{T}\left(\lambda_{1} A_{u_{2}}+\lambda_{2} B_{v_{1}}\right) \mathrm{x}<\alpha \lambda_{1}-\beta \lambda_{2}, \\
\frac{1}{2} x^{T}\left(\lambda_{1} A_{u_{2}}+\lambda_{2} B_{v_{2}}\right) \mathrm{x}<\alpha \lambda_{1}-\beta \lambda_{2} .
\end{array}\right.
$$

$$
\Lambda=\left\{\left(\begin{array}{c}
\frac{1}{2} x^{T}\left(\lambda_{1} A_{u_{1}}+\lambda_{2} B_{v_{1}}\right) x, \frac{1}{2} x^{T}\left(\lambda_{1} A_{u_{1}}+\lambda_{2} B_{v_{2}}\right) x, \\
\frac{1}{2} x^{T}\left(\lambda_{1} A_{u_{2}}+\lambda_{2} B_{v_{1}}\right) x, \frac{1}{2} x^{T}\left(\lambda_{1} A_{u_{2}}+\lambda_{2} B_{v_{2}}\right) x \mid x \in \mathbb{R}^{n}
\end{array}\right)\right\} .
$$

Let us consider the following mapping:

$\Phi: \mathbb{R}^{4} \rightarrow \mathbb{R}^{4},\left(t_{1}, t_{2}, t_{3}, t_{4}\right) \mapsto\left(\lambda_{1} t_{1}+\lambda_{2} t_{3}, \lambda_{1} t_{1}+\lambda_{2} t_{4}, \lambda_{1} t_{2}+\lambda_{2} t_{3}, \lambda_{1} t_{2}+\lambda_{2} t_{4}\right)$.

$\Phi$ is a linear transformation and $\Omega$ is a convex set (assumption), it follows that $\Lambda=\Phi(\Omega)$ is a convex set, see Equation (1) in Theorem 2.

By remarking that $\left(\left(\alpha \lambda_{1}-\beta \lambda_{2}\right),\left(\alpha \lambda_{1}-\beta \lambda_{2}\right),\left(\alpha \lambda_{1}-\beta \lambda_{2}\right),\left(\alpha \lambda_{1}-\beta \lambda_{2}\right)\right) \notin \Lambda+$ int $\mathbb{R}_{+}^{4}$, the hyperplane separation theorem shows that there are $\left(\gamma_{i}\right)_{1 \leq i \leq 4} \in \mathbb{R}_{+}^{4} \backslash\left\{0_{\mathbb{R}^{4}}\right\}$ such that for all $x \in \mathbb{R}^{n}$, one has:

$$
\begin{aligned}
& \gamma_{1}\left[\frac{1}{2} x^{T}\left(\lambda_{1} A_{u_{1}}+\lambda_{2} B_{v_{1}}\right) x-\left(\alpha \lambda_{1}-\beta \lambda_{2}\right)\right]+ \\
& \gamma_{2}\left[\frac{1}{2} x^{T}\left(\lambda_{1} A_{u_{1}}+\lambda_{2} B_{v_{2}}\right) x-\left(\alpha \lambda_{1}-\beta \lambda_{2}\right)\right]+ \\
& \gamma_{3}\left[\frac{1}{2} x^{T}\left(\lambda_{1} A_{u_{2}}+\lambda_{2} B_{v_{1}}\right) x-\left(\alpha \lambda_{1}-\beta \lambda_{2}\right)\right]+ \\
& \gamma_{4}\left[\frac{1}{2} x^{T}\left(\lambda_{1} A_{u_{2}}+\lambda_{2} B_{v_{2}}\right) x-\left(\alpha \lambda_{1}-\beta \lambda_{2}\right)\right] \geq 0 .
\end{aligned}
$$


Put $\gamma=\gamma_{1}+\gamma_{2}+\gamma_{3}+\gamma_{4}$. Then, we have the following equivalences:

$$
\begin{aligned}
(7) \Leftrightarrow & \lambda_{1}\left[\frac{1}{2} x^{T}\left(\gamma_{1} A_{u_{1}}+\gamma_{2} A_{u_{1}}+\gamma_{3} A_{u_{2}}+\gamma_{4} A_{u_{2}}\right) x-\alpha \gamma\right] \\
+ & \lambda_{2}\left[\frac{1}{2} x^{T}\left(\gamma_{1} B_{v_{1}}+\gamma_{2} B_{v_{2}}+\gamma_{3} B_{v_{1}}+\gamma_{4} B_{v_{2}}\right) x+\beta \gamma\right] \geq 0 \\
\Leftrightarrow & \lambda_{1}\left[\frac{1}{2} x^{T}\left(\left(\gamma_{1}+\gamma_{2}\right) A_{u_{1}}+\left(\gamma_{3}+\gamma_{4}\right) A_{u_{2}}\right) \mathrm{x}-\alpha \gamma\right] \\
+ & \lambda_{2}\left[\frac{1}{2} x^{T}\left(\left(\gamma_{1}+\gamma_{3}\right) B_{v_{1}}+\left(\gamma_{2}+\gamma_{4}\right) B_{v_{2}}\right) \mathrm{x}+\beta \gamma\right] \geq 0 \\
\Leftrightarrow & \lambda_{1}\left[\frac { 1 } { 2 } x ^ { T } \left(\left(\gamma_{1}+\gamma_{2}\right)\left(A_{1}+u_{1} A_{2}\right)+\right.\right. \\
& \left.\left(\gamma-\gamma_{1}-\gamma_{2}\right)\left(\left(A_{1}+u_{2} A_{2}\right)\right) \mathrm{x}-\alpha \gamma\right] \\
+ & \lambda_{2}\left[\frac { 1 } { 2 } x ^ { T } \left(\left(\gamma_{1}+\gamma_{3}\right)\left(B_{1}+v_{1} B_{2}\right)+\right.\right. \\
& \left.\left.\left(\gamma-\gamma_{1}-\gamma_{3}\right)\left(B_{1}+v_{2} B_{2}\right)\right) \mathrm{x}+\beta \gamma\right] \geq 0 \\
\Leftrightarrow & \lambda_{1}\left[\frac { 1 } { 2 } x ^ { T } \left(\left(\gamma_{1}+\gamma_{2}\right) u_{1} A_{2}+\gamma\left(A_{1}+u_{2} A_{2}\right)\right.\right. \\
& \left.\left.-\left(\gamma_{1}+\gamma_{2}\right) u_{2} A_{2}\right) \mathrm{x}-\alpha \gamma\right] \\
+ & \lambda_{2}\left[\frac { 1 } { 2 } x ^ { T } \left(\left(\gamma_{1}+\gamma_{3}\right) v_{1} B_{2}+\gamma\left(B_{1}+v_{2} B_{2}\right)\right.\right. \\
+ & \left.\left.\left(\gamma_{1}+\gamma_{3}\right) v_{2} B_{2}\right) \mathrm{x}+\beta \gamma\right] \geq 0 \\
\Leftrightarrow & \lambda_{1}\left[\frac{1}{2} x^{T}\left(A_{1}+u_{2} A_{2}+\frac{\left(\gamma_{1}+\gamma_{2}\right)\left(u_{1}-u_{2}\right) A_{2}}{\gamma}\right) \mathrm{x}-\alpha\right] \\
+ & \lambda_{2}\left[\frac{1}{2} x^{T}\left(B_{1}+v_{2} B_{2}+\frac{\left(\gamma_{1}+\gamma_{3}\right)\left(v_{1}-v_{2}\right) B_{2}}{\gamma}\right) \mathrm{x}+\beta\right] \geq 0 \\
\Leftrightarrow & \lambda_{1}\left[\frac{1}{2} x^{T}\left(A_{1}+\frac{\gamma u_{2}+\left(\gamma_{1}+\gamma_{2}\right)\left(u_{1}-u_{2}\right)}{\gamma} A_{2}\right) \mathrm{x}-\alpha\right] \\
+ & \lambda_{2}\left[\frac{1}{2} x^{T}\left(B_{1}+\frac{\gamma v_{2}+\left(\gamma_{1}+\gamma_{3}\right)\left(v_{1}-v_{2}\right)}{\gamma} B_{2}\right) \mathrm{x}+\beta\right] \geq 0 \\
\Leftrightarrow & \lambda_{1}\left[\frac{1}{2} x^{T}\left(A_{1}+\frac{\left(\gamma_{1}+\gamma_{2}\right) u_{1}+\left(\gamma_{3}+\gamma_{4}\right) u_{2}}{\gamma} A_{2}\right) \mathrm{x}-\alpha\right] \\
+ & \lambda_{1}\left[\frac{1}{2} x^{T}\left(B_{1}+\frac{\left(\gamma_{1}+\gamma_{3}\right) v_{1}+\left(\gamma_{3}+\gamma_{4}\right) v_{2}}{\gamma} B_{2}\right) \mathrm{x}+\beta\right] \geq 0
\end{aligned}
$$

Consequently, we obtain the existence of $(\bar{u}, \bar{v}) \in \mathcal{U}_{1} \times \mathcal{U}_{2}$ et $\left(\lambda_{1}, \lambda_{2}\right) \in \mathbb{R}_{+}^{2} \backslash\{(0,0)\}$ such that:

$\lambda_{1}\left(\frac{1}{2} x^{T} A_{\bar{u}} \mathrm{x}-\alpha\right)+\lambda_{2}\left(\frac{1}{2} x^{T} B_{\bar{v}} \mathrm{x}+\beta\right) \geq 0, \forall x \in \mathbb{R}^{n}$

Where:

$$
\bar{u}=\frac{\left(\gamma_{1}+\gamma_{2}\right)}{\gamma} u_{1}+\frac{\left(\gamma_{3}+\gamma_{4}\right)}{\gamma} u_{2} \text { and } \bar{v}=\frac{\left(\gamma_{1}+\gamma_{3}\right)}{\gamma} v_{1}+\frac{\left(\gamma_{2}+\gamma_{4}\right)}{\gamma} v_{2}
$$

Remark 1 Some particular cases of Theorem 2 are given as, if :

$-U_{1}$ or $U_{2}$ is a singleton, then Theorem 2 is reduced to Theorem 3.1 of [31].

- Both $U_{1}$ and $U_{2}$ are singletons, then Theorem 2 consists merely of Dines Theorem (see Theorem 1 or [16]). 
Note that the following result is an uncertain version of the classical S-lemma given in Lemma 1.

\section{Corollary 1 (Robust $\mathbf{S}$-lemma)}

Let $A_{0}, A_{1}, B_{0}, B_{1} \in \mathbb{S}_{n}(\mathbb{R}) ; u_{1}, u_{2}, v_{1}, v_{2} \in \mathbb{R}$ such that $u_{1} \leq u_{2}$ and $v_{1} \leq v_{2}$. Assume that: $\Omega=\left\{\left(x^{T} A_{u_{1}} x, x^{T} A_{u_{2}} x, x^{T} B_{v_{1}} x, x^{T} B_{v_{2}} x\right) \mid x \in \mathbb{R}^{n}\right\}$ is convex.

There exists $x_{0} \in \mathbb{R}^{n}$ such that $x_{0}^{T} B_{v} x_{0}<0$ for all $\mathrm{v} \in \mathcal{U}_{2}$.

Then, the following statements are equivalent:

(i) $x^{T} B_{v} \mathrm{x} \leq 0, \forall v \in \mathcal{U}_{2} \Rightarrow x^{T} A_{u} \mathrm{x} \geq 0, \forall u \in \mathcal{U}_{1}$.

(ii) $\exists \lambda \geq 0, \exists(\bar{u}, \bar{v}) \in \mathcal{U}_{1} \times \mathcal{U}_{2} \mid A_{\bar{u}}+\lambda B_{\bar{v}} \geqslant 0$.

Proof $[(i i) \Longrightarrow(i)]$. This implication is always satisfied.

$[(\mathrm{i}) \Rightarrow$ (ii)]. Suppose that (i) holds. Hence, the inequalities system

$$
\left\{\begin{array}{l}
x^{T} A_{u} \mathrm{x}<0, \forall u \in \mathcal{U}_{1} \\
x^{T} B_{v} \mathrm{x}<0, \forall v \in \mathcal{U}_{2}
\end{array}\right.
$$

has no solution. From Theorem 2 , there exist $\left(\lambda_{1}, \lambda_{2}\right) \in \mathbb{R}_{+}^{2} \backslash\{(0,0)\}$,

$(\bar{u}, \bar{v}) \in \mathcal{U}_{1} \times \mathcal{U}_{2}$ such that:

$$
\lambda_{1} x^{T} A_{\bar{u}} \mathrm{x}+\lambda_{2} x^{T} B_{\bar{v}} \mathrm{x} \geq 0, \forall x \in \mathbb{R}^{n} .
$$

If $\lambda_{1}=0$ then $\lambda_{1}>0$ and $x^{T} B_{\bar{v}} \mathrm{x} \geq 0$ for all $\in \mathbb{R}^{n}$. Which is a contradiction since $x_{0}^{T} B_{v} x_{0}<$ 0 Hence $\lambda_{1}>0$. It follows that $A_{\bar{u}}+\lambda B_{\bar{v}} \geqslant 0$, with: $\lambda=\frac{\lambda_{2}}{\lambda_{1}}$.

\section{Characterization of Robust Solution of QCQOP under Data Uncertainty}

\subsection{Homogeneous Case}

In this subsection, we give a characterization of robust optimal solution for homogeneous quadratic programming problem under data uncertainty.

For this purpose, we consider the following problem

$$
\begin{aligned}
\min _{x \in \mathbb{R}^{n}} & \frac{1}{2} x^{T} A_{u} \mathrm{x}, \\
\text { s.t. } & \frac{1}{2} x^{T} B_{v} \mathrm{x}+\beta \leq 0 .
\end{aligned}
$$

The robust counterpart (worst case) of (H-QCQP) is given by

$$
\begin{aligned}
\min _{x \in \mathbb{R}^{n}} & \max _{\boldsymbol{u} \in \mathcal{U}_{1}} \frac{1}{2} x^{T} A_{u} \mathrm{x}, \quad \text { (RH-QCQP) } \\
\text { s.t. } & \max _{\boldsymbol{v} \in \mathcal{U}_{2}} \frac{1}{2} x^{T} B_{v} \mathrm{x}+\beta \leq 0 .
\end{aligned}
$$

\section{Theorem 3 (Characterization of robust solution)}

Let $A_{0}, A_{1}, B_{0}, B_{1} \in \mathbb{S}_{n}(\mathbb{R}) ; u_{1}, u_{2}, v_{1}, v_{2} \in \mathbb{R}$ such that $u_{1} \leq u_{2}$ and $v_{1} \leq v_{2}$. Assume that:

$\left(H_{1}\right)$ There exists $x_{0} \in \mathbb{R}^{n}$ such that $x_{0}^{T} B_{v} x_{0}+\beta<0$ for all $\mathrm{v} \in \mathcal{U}_{2}$.

$\left(H_{2}\right)$ The set $\Omega=\left\{\left(x^{T} A_{u_{1}} x, x^{T} A_{u_{2}} x, x^{T} B_{v_{1}} x, x^{T} B_{v_{2}} x\right) \mid x \in \mathbb{R}^{n}\right\}$ is convex. 
Then, the following statements are equivalent:

(i) $\bar{x}$ is a robust optimal solution of $(\mathrm{H}-\mathrm{QCQP})$;

(ii) there exist $\lambda \geq 0$ and $(\bar{u}, \bar{v}) \in \mathcal{U}_{1} \times \mathcal{U}_{2}$ such that:

$$
\begin{aligned}
& \left(A_{\bar{u}}+\lambda B_{\bar{v}}\right) \bar{x}=0, \quad \text { (First-order Condition). } \\
& \left(\frac{1}{2} \bar{x}^{T} B_{\bar{v}} \bar{x}+\beta\right)=0, \quad \text { (Complementary Slackness) } \\
& A_{\bar{u}}+\lambda B_{\bar{v}} \geqslant 0,
\end{aligned}
$$

Proof $[(i i) \Longrightarrow(i)]$. Assume that $\bar{x}$ is a robust optimal solution of (H-QCQP).

Then, for all $x \in \mathbb{R}^{n}$ :

$\frac{1}{2} x^{T} B_{v} \mathrm{x}+\beta \leq 0, \forall \mathrm{v} \in \mathcal{U}_{2} \Rightarrow \max _{\boldsymbol{u} \in \mathcal{U}_{1}} \frac{1}{2} x^{T} A_{u} \mathrm{x} \geq \alpha:=\max _{\boldsymbol{u} \in \mathcal{U}_{1}} \frac{1}{2} \bar{x}^{T} A_{u} \bar{x}$.

This implies that

$$
\begin{aligned}
& \max _{\boldsymbol{u} \in \mathcal{U}_{1}} \frac{1}{2} x^{T} A_{u} \mathrm{x}<\alpha, \\
& \frac{1}{2} x^{T} B_{v} \mathrm{x}+\beta \leq 0, \forall \mathrm{v} \in \mathcal{U}_{2}
\end{aligned}
$$

has no solution. This means that the following inequalities system

$$
\left\{\begin{array}{l}
\frac{1}{2} x^{T} A_{u} \mathrm{x}<\alpha, \forall \mathrm{u} \in \mathcal{U}_{1}, \\
\frac{1}{2} x^{T} B_{v} \mathrm{x}+\beta \leq 0, \forall \mathrm{v} \in \mathcal{U}_{2},
\end{array}\right.
$$

has no solution. Thanks to hypothesis (H2) by applying Theorem 2 , there exist

$\left(\lambda_{1}, \lambda_{2}\right) \in \mathbb{R}_{+}^{2} \backslash\{(0,0)\}$ and $(\bar{u}, \bar{v}) \in \mathcal{U}_{1} \times \mathcal{U}_{2}$ such that:

$\lambda_{1}\left(\frac{1}{2} x^{T} A_{\bar{u}} \mathrm{x}-\alpha\right)+\lambda_{2}\left(\frac{1}{2} x^{T} B_{\bar{v}} \mathrm{x}+\beta\right) \geq 0, \forall x \in \mathbb{R}^{n}$.

If $\lambda_{1}=0$, then: $\lambda_{2}\left(\frac{1}{2} x^{T} B_{\bar{v}} \mathrm{x}+\beta\right) \geq 0$ for all $x \in \mathbb{R}^{n}$.

For $\mathrm{x}=x_{0}$, with $\left(H_{1}\right)$, we see that $\lambda_{1} \leq 0$ and $\lambda_{2}=0$, which contradicts the fact that $\left(\lambda_{1}, \lambda_{2}\right) \in$ $\mathbb{R}_{+}^{2} \backslash\{(0,0)\}$. Hence, $\lambda_{1}<0$. This means that

$$
\left(\frac{1}{2} x^{T} A_{\bar{u}} \mathrm{x}-\alpha\right)+\lambda\left(\frac{1}{2} x^{T} B_{\bar{v}} \mathrm{x}+\beta\right) \geq 0, \forall x \in \mathbb{R}^{n}
$$

Where $\lambda=\frac{\lambda_{2}}{\lambda_{1}}$. Inequality (11) implies that

$$
\left(\max _{\boldsymbol{u} \in \mathcal{U}_{1}} \frac{1}{2} x^{T} A_{u} \mathrm{x}-\alpha\right)+\lambda\left(\frac{1}{2} x^{T} B_{\bar{v}} \mathrm{x}+\beta\right) \geq 0, \forall x \in \mathbb{R}^{n}
$$

It is clear that for $x=\bar{x}$, one has :

$$
\left(\frac{1}{2} \bar{x}^{T} B_{\bar{v}} \bar{x}+\beta\right)=0 \text { (by using the definition of in Inequality (10)). }
$$

Then, the complementary slackness conditions hold. Let us consider the function defined by:

$\varphi: \mathbb{R}^{n} \rightarrow \mathbb{R}, \mathrm{x} \mapsto \varphi(\mathrm{x})=\left(\frac{1}{2} x^{T} A_{\bar{u}} \mathrm{x}-\alpha\right)+\lambda\left(\frac{1}{2} x^{T} B_{\bar{v}} \mathrm{x}+\beta\right)$.The Inequality (11) implies that $\varphi(\mathrm{x}) \geq 0$ and $\varphi(\bar{x}) \geq 0 . \bar{x}$ minimize $\varphi$ on $\mathbb{R}^{n}$ and $\varphi$ is twice differentiable. Then, $\bar{x}$ solve the Euler equation $\nabla \varphi(\mathrm{x})=0$ and inequalities system $\nabla^{2} \varphi(\mathrm{x}) \geqslant 0$. Thus, the first and second order necessary conditions of optimality in the point $\bar{x}$ lead to: 


$$
\left\{\begin{array}{lc}
\left(A_{\bar{u}}+\lambda B_{\bar{v}}\right) \bar{x}=0, & \text { (First-order Condition) } . \\
A_{\bar{u}}+\lambda B_{\bar{v}} \geqslant 0, & \text { (Second-order Condition) } .
\end{array}\right.
$$

$[(i i)=)(\mathrm{i})]$. Now, suppose that there exist $\lambda \geq 0$ and $(\bar{u}, \bar{v}) \in \mathcal{U}_{1} \times \mathcal{U}_{2}$ such that:

$$
\left\{\begin{array}{lc}
\left(A_{\bar{u}}+\lambda B_{\bar{v}}\right) \bar{x}=0, & \text { (First-order Condition) } \\
\lambda\left(\frac{1}{2} \bar{x}^{T} B_{\bar{v}} \bar{x}+\beta\right)=0, & \text { (Complementary Slackness) } \\
A_{\bar{u}}+\lambda B_{\bar{v}} \geqslant 0, & \text { (Second-order Condition) } .
\end{array}\right.
$$

Let $\mathrm{x}$ be a robust feasible solution of (H-QCQP). By using complementary slackness condition, one has:

$$
\frac{1}{2} x^{T} B_{\bar{v}} x+\beta \leq \lambda\left(\frac{1}{2} \bar{x}^{T} B_{\bar{v}} \bar{x}+\beta\right) \text {. }
$$

Let us consider $\mathbb{R}^{n} \rightarrow \mathbb{R}, \mathrm{x} \mapsto \varphi(\mathrm{x}):=\frac{1}{2} x^{T} A_{\bar{u}} \mathrm{x}+\lambda\left(\frac{1}{2} x^{T} B_{\bar{v}} \mathrm{x}+\beta\right)$.

The first-order condition and second-order condition (necessary condition of optimality) mean that $\nabla \varphi(\bar{x})=0$ and $\varphi$ is convex function over $\mathbb{R}^{n}$. Thus,

$$
\begin{aligned}
\varphi(\mathrm{x}) \geq \varphi(\bar{x}) & \Rightarrow \frac{1}{2} x^{T} A_{\bar{u} \mathrm{x}} \geq \frac{1}{2} \bar{x}^{T} A_{\bar{u}} \bar{x}+\lambda\left(\frac{1}{2} \bar{x}^{T} B_{\bar{v}} \bar{x}+\beta\right)- \\
& \lambda\left(\frac{1}{2} x^{T} B_{\bar{v}} \mathrm{x}+\beta\right) \\
\Rightarrow & \frac{1}{2} x^{T} A_{\bar{u}} \mathrm{x} \geq \frac{1}{2} \bar{x}^{T} A_{\bar{u}} \bar{x}-\lambda\left(\frac{1}{2} x^{T} B_{\bar{v}} \mathrm{x}+\beta\right) \\
\Rightarrow & \frac{1}{2} x^{T} A_{\bar{u}} \mathrm{x} \geq \frac{1}{2} \bar{x}^{T} A_{\bar{u}} \bar{x} .
\end{aligned}
$$

Hence, $\bar{x}$ is robust optimal solution of (H-QCQP).

Remark 2 Note that the robust optimal solution characterization of the uncertain homogeneous quadratic optimization problem given in Theorem 3 was established in [31] by assuming that $\mathcal{U}_{1}$ is a singleton and where the corresponding additive scalar is assumed to be negative (regarding to our problem formulation).

\subsection{Non-homogeneous Case}

In this subsection, at first we will derive necessary and sufficient conditions of robust optimal solution for quadratic programming under bounded interval uncertainty. Secondly, we will give a characterization of optimal solutions of the optimistic dual.

Let us consider the following uncertain quadratic problem

$$
\begin{aligned}
& \min _{x \in \mathbb{R}^{n}} \frac{1}{2} x^{T} A_{u} \mathrm{x}+a_{\omega}^{T} \mathrm{x} \\
& \text { s.t. } \frac{1}{2} x^{T} B_{v} \mathrm{x}+b_{\varsigma}^{T} \mathrm{x}+\beta
\end{aligned}
$$

where $A_{u}, B_{v} \in \mathbb{S}_{n}(\mathbb{R}), a_{\omega}, b_{\varsigma} \in \mathbb{R}^{n}, \beta \in \mathbb{R}$ and $(\mathrm{u}, \mathrm{v}, \omega, \varsigma)$ belongs to the uncertainty set $\mathcal{U}=\prod_{i=1}^{4} \mathcal{U}_{i}=\left[u_{1}, u_{2}\right] \times\left[v_{1}, v_{2}\right] \times\left[\omega_{1}, \omega_{2}\right] \times\left[\varsigma_{1}, \varsigma_{2}\right]$ 
The robust counterpart (worst case) of (NH-QCQP) is given by

$$
\begin{aligned}
& \min _{x \in \mathbb{R}^{n}} \max _{\boldsymbol{u} \in \mathcal{U}_{1}} \frac{1}{2} x^{T} A_{u} \mathrm{x}+\max _{\boldsymbol{\omega} \in \mathcal{U}_{3}} a_{\omega}^{T} \mathrm{x}, \\
& \text { s.t. } \max _{\boldsymbol{v} \in \mathcal{U}_{2}} \frac{1}{2} x^{T} B_{v} \mathrm{x}+\max _{\boldsymbol{\mathbf { a }} \in \mathcal{U}_{4}} b_{\zeta}^{T} \mathrm{x}+\beta \leq 0 .
\end{aligned}
$$

(RHN-QCQP)

Given a $\bar{x}$ in $\mathbb{R}^{n}$, we set $\alpha=-\max _{(u, \omega) \in \mathcal{U}_{1} \times \mathcal{U}_{3}}\left\{\frac{1}{2} \bar{x}^{T} A_{u} \bar{x}+a_{\omega}^{T} \bar{x}\right\}$ and

$\Xi_{\bar{x}}=\left\{\left(\begin{array}{l}x \\ t\end{array}\right)^{T} H_{1}\left(\begin{array}{l}x \\ t\end{array}\right),\left(\begin{array}{c}x \\ t\end{array}\right)^{T} H_{2}\left(\begin{array}{c}x \\ t\end{array}\right),\left(\begin{array}{c}x \\ t\end{array}\right)^{T} H_{3}\left(\begin{array}{c}x \\ t\end{array}\right),\left(\begin{array}{c}x \\ t\end{array}\right)^{T} H_{4}\left(\begin{array}{c}x \\ t\end{array}\right) \mid(x, t) \in \mathbb{R}^{n} \times \mathbb{R}\right\}$,

Where:

$H_{1}=\left(\begin{array}{cc}A_{u_{1}} & a_{\omega_{1}} \\ a_{\omega_{1}}^{T} & 2 \alpha\end{array}\right), H_{2}=\left(\begin{array}{cc}A_{u_{2}} & a_{\omega_{2}} \\ a_{\omega_{2}}^{T} & 2 \alpha\end{array}\right), H_{3}=\left(\begin{array}{cc}B_{v_{1}} & b_{\varsigma_{1}} \\ b_{\zeta_{1}}^{T} & 2 \beta\end{array}\right)$ and $H_{4}=\left(\begin{array}{cc}B_{v_{2}} & b_{\varsigma_{2}} \\ b_{\varsigma_{2}}^{T} & 2 \beta\end{array}\right)$

We give the following definition with regards to convexity of QCQOPs at a given point in $\mathbb{R}^{n}$. This definition is inspired by the works of Dines (1941), Polyak (1998) and Jeyakumar (2012) about analysis of convexity properties of quadratic forms, and named after them.

Definition 1 (Convexity in the sense of Dines-Polyak-Jeyakumar) The problem (NH-QCQP) is said to be DPJ-convex (or regular) w.r.t. $\quad \bar{x}$ if the set $\Xi_{\bar{x}}$ is convex.

\section{Theorem 3 (Characterization of robust solution)}

Let $\bar{x}$ be a robust feasible solution of (NH-QCQP) such that;

$\left(H_{1}\right) \quad$ (NH-QCQP) is DPJ-convex at $\bar{x}$;

$\left(H_{2}\right)$ there exists $x_{0} \in \mathbb{R}^{n}$ such that $x_{0}^{T} B_{v} x_{0}+b_{\varsigma}^{T} x_{0}+\beta<0$ for all $(\mathrm{v}, \varsigma) \in \mathcal{U}_{2} \times \mathcal{U}_{4}$.

Then, the following statements are equivalent:

(i) $\bar{x}$ is a robust optimal solution of (NH-QCQP);

(ii) there exist $\lambda \geq 0$ and $(\bar{u}, \bar{v}, \bar{\omega}, \bar{\varsigma}) \in \mathcal{U}$ such that:

$$
\left\{\begin{array}{lc}
\left(A_{\bar{u}}+\lambda B_{\bar{v}}\right) \bar{x}+a_{\bar{\omega}}+\lambda b_{\bar{\varsigma}}=0, & \text { (First-order Condition) } \\
\lambda\left(\frac{1}{2} \bar{x}^{T} B_{\bar{v}} \bar{x}+b_{\bar{\varsigma}}+\beta\right)=0, & \text { (Complementary Slackness) } \\
A_{\bar{u}}+\lambda B_{\bar{v}} \geqslant 0 . & \text { (Second-order Condition) } .
\end{array}\right.
$$

Proof $[(i i) \Rightarrow(i)]$. Let $\bar{x}$ be a robust optimal solution of

(NH-QCQP) and $\alpha=-\max _{(u, \omega) \in \mathcal{U}_{1} \times u_{3}}\left\{\frac{1}{2} \bar{x}^{T} A_{u} \bar{x}+a_{\omega}^{T} \bar{x}\right\}$.

Then for all $\mathrm{x} \in \mathbb{R}^{n}$, one has:

$$
\begin{aligned}
& \max _{(v, \varsigma) \in \mathcal{U}_{2} \times \mathcal{U}_{4}}\left\{\frac{1}{2} x^{T} B_{v} \mathrm{x}+b_{\varsigma}^{T} \mathrm{x}\right\}+\beta \leq 0 \\
& \Rightarrow \max _{(u, \omega) \in \mathcal{U}_{1} \times \mathcal{U}_{3}}\left\{\frac{1}{2} x A_{u} x+a_{\omega}^{T} x\right\} . \geq-\alpha .
\end{aligned}
$$


Consequently, the system

$$
\left\{\begin{array}{c}
\max _{(u, \omega) \in \mathcal{U}_{1} \times \mathcal{U}_{3}}\left\{\frac{1}{2} x A_{u} x+a_{\omega}^{T} x\right\} .<-\alpha \\
\max _{(v, \zeta) \in \mathcal{U}_{2} \times u_{4}}\left\{\frac{1}{2} x^{T} B_{v} \mathrm{x}+b_{\varsigma}^{T} \mathrm{x}\right\}+\beta<0
\end{array}\right.
$$

has no solution. Thus, the system

has no solution.

$$
\begin{aligned}
& \frac{1}{2} x^{T} A_{u} \mathrm{x}+a_{\omega}^{T} \mathrm{x}+\alpha<0, \forall(\mathrm{u}, \omega) \in \mathcal{U}_{1} \times \mathcal{U}_{3} \\
& \frac{1}{2} x^{T} B_{v} \mathrm{x}+b_{\varsigma}^{T} \mathrm{x}+\beta<0, \quad \forall(\mathrm{v}, \varsigma) \in \mathcal{U}_{2} \times \mathcal{U}_{4}
\end{aligned}
$$

Moreover, we can show that for all $t \in \mathbb{R}$, the following system

$$
\left\{\begin{array}{l}
\frac{1}{2} x^{T} A_{u} \mathrm{x}+t a_{\omega}^{T} \mathrm{x}+t^{2} \alpha<0, \forall(\mathrm{u}, \omega) \in \mathcal{U}_{1} \times \mathcal{U}_{3}, \\
\frac{1}{2} x^{T} B_{v} \mathrm{x}+t b_{\varsigma}^{T} \mathrm{x}+t^{2} \beta<0, \forall(\mathrm{v}, \varsigma) \in \mathcal{U}_{2} \times \mathcal{U}_{4},
\end{array}\right.
$$

has no solution in $\mathbb{R}^{n} \times \mathbb{R}$. Indeed if $\left(x^{*}, t^{*}\right) \in \mathbb{R}^{n} \times \mathbb{R}$ is a solution of $\left(S_{2}\right)$, then

$$
\left\{\begin{array}{l}
\frac{1}{2} x^{*^{T}} A_{u} x^{*}+t^{*} a_{\omega}^{T} x^{*}+t^{*^{2}} \alpha<0, \forall(\mathrm{u}, \omega) \in \mathcal{U}_{1} \times \mathcal{U}_{3} \\
\frac{1}{2} x^{*^{T}} B_{v} x^{*}+t^{*} b_{\varsigma}^{T} x^{*}+t^{*^{2}} \beta<0, \forall(\mathrm{v}, \varsigma) \in \mathcal{U}_{2} \times \mathcal{U}_{4}
\end{array}\right.
$$

So, for $t^{*} \neq 0$, one has:

$$
\left\{\begin{array}{l}
\frac{1}{2}\left(\frac{x^{*}}{t^{*}}\right)^{T} A_{u}\left(\frac{x^{*}}{t^{*}}\right)+a_{\omega}^{T}\left(\frac{x^{*}}{t^{*}}\right)+\alpha<0, \forall(\mathrm{u}, \omega) \in \mathcal{U}_{1} \times \mathcal{U}_{3} \\
\frac{1}{2}\left(\frac{x^{*}}{t^{*}}\right)^{T} B_{v}\left(\frac{x^{*}}{t^{*}}\right)+b_{\varsigma}^{T}\left(\frac{x^{*}}{t^{*}}\right)+\beta<0, \forall(\mathrm{v}, \varsigma) \in \mathcal{U}_{2} \times \mathcal{U}_{4}
\end{array}\right.
$$

Which contradicts the fact that $\left(S_{1}\right)$ has no solution.

If $t^{*}=0$, it follows that $x^{*}$ is a solution of the system

$$
\left\{\begin{array}{l}
\frac{1}{2} x^{T} A_{u} \mathrm{x}<0, \forall \mathrm{u} \in \mathcal{U}_{1} \\
\frac{1}{2} x^{T} B_{v} \mathrm{x}<0, \forall \mathrm{v} \in \mathcal{U}_{2}
\end{array}\right.
$$

In this case $x^{*}=0$ and for a fairly large number $\mathrm{n} \in \mathbb{N}, x_{n}=\mathrm{n} x^{*}$ is a solution of the system $\left(S_{1}\right)$. Which is a contradiction. We conclude that the system $\left(S_{2}\right)$ has no solution.

Let us show that there exist $(\bar{u}, \bar{v}, \bar{\omega}, \bar{\varsigma}) \in \mathcal{U}$ and $\left(\lambda_{1}, \lambda_{2}\right) \in \mathbb{R}_{+}^{2} \backslash\{(0,0)\}$ such that

$\lambda_{1}\left(\frac{1}{2} x^{T} A_{\bar{u}} x+a T_{\bar{\omega}}^{T} x+\alpha\right)+\lambda_{2}\left(\frac{1}{2} x^{T} B_{\bar{v}} x+b_{\bar{\varsigma}}{ }^{T} x+\beta\right) \geq 0, \forall x \in \mathbb{R}^{n}$.

One proceeds by distinguishing four cases as follows. 
First case : $u_{1}<u_{2}$ and $v_{1}<v_{2}$.

Let

$M_{1}=\left(\begin{array}{cc}A_{1} & a_{1}+\frac{\omega_{1} u_{2}-\omega_{2} u_{1}}{u_{2}-u_{1}} a_{2} \\ a_{1}^{T}+\frac{\omega_{1} u_{2}-\omega_{2} u_{1}}{u_{2}-u_{1}} a_{2}{ }^{T} & 2 \alpha\end{array}\right)$

$M_{2}=\left(\begin{array}{cc}A_{2} & \frac{\omega_{2}-\omega_{1}}{u_{2}-u_{1}} a_{2} \\ \frac{\omega_{2}-\omega_{1}}{u_{2}-u_{1}} a_{2}^{T} & 0\end{array}\right)$,

$M_{3}=\left(\begin{array}{cc}B_{1} & b_{1}+\frac{\omega_{1} u_{2}-\omega_{2} u_{1}}{u_{2}-u_{1}} b_{1} \\ b_{1}^{T}+\frac{\varsigma_{1} v_{2}-\varsigma_{2} v_{1}}{v_{2}-v_{1}} b_{1}{ }^{T} & 2 \beta\end{array}\right)$,

and $\quad M_{4}=\left(\begin{array}{cc}B_{2} & \frac{\varsigma_{2}-\varsigma_{1}}{v_{2}-v_{1}} b_{2} \\ \frac{\varsigma_{2}-\varsigma_{1}}{v_{2}-v_{1}} b_{2}{ }^{T} & 0\end{array}\right)$.

Then, we have the following linear matrix equalities :

$$
\begin{aligned}
& M_{1}+u_{1} M_{2}=H_{1} ; \quad M_{1}+u_{2} M_{2}=H_{2} ; \\
& M_{3}+v_{1} M_{4}=H_{3} \text { and } M_{3}+v_{2} M_{4}=H_{4} .
\end{aligned}
$$

By applying the fact that $\left(S_{1}\right)$ has no solution, we deduce that the system below

$$
\left\{\begin{array}{l}
\frac{1}{2}\left(\begin{array}{l}
x \\
t
\end{array}\right)^{T}\left(M_{1}+u M_{2}\right)\left(\begin{array}{l}
x \\
t
\end{array}\right)<0, \forall \mathrm{u} \in \mathcal{U}_{1}, \\
\frac{1}{2}\left(\begin{array}{l}
x \\
t
\end{array}\right)^{T}\left(M_{1}+v M_{2}\right)\left(\begin{array}{l}
x \\
t
\end{array}\right)<0, \forall \mathrm{v} \in \mathcal{U}_{2},
\end{array}\right.
$$

also has no solution.

Thanks to the convexity of the set $\Xi_{\bar{x}}$ and from Theorem 2 there exist $\left(\lambda_{1}, \lambda_{2}\right) \in \mathbb{R}_{+}^{2} \backslash\{(0,0)\}(\bar{u}, \bar{v}) \in \mathcal{U}_{1} \times \mathcal{U}_{2}$ such that

$\lambda_{1}\left[\frac{1}{2}\left(\begin{array}{l}x \\ t\end{array}\right)^{T}\left(M_{1}+\bar{u} M_{2}\right)\left(\begin{array}{l}x \\ t\end{array}\right)\right]+$

$\lambda_{2}\left[\frac{1}{2}\left(\begin{array}{c}x \\ t\end{array}\right)^{T}\left(M_{3}+\bar{v} M_{4}\right)\left(\begin{array}{l}x \\ t\end{array}\right)\right] \geq 0$

For all $(x, t) \in \mathbb{R}^{n} \times \mathbb{R}$. 
By setting $\mathrm{t}=1$, one has :

$\lambda_{1}\left[\frac{1}{2} x^{T} A_{\bar{u}} x+\left(a_{1}+\left(\frac{\omega_{1} u_{2}-\omega_{2} u_{1}}{u_{2}-u_{1}}\right)+\bar{u} \frac{\omega_{1} u_{2}-\omega_{2} u_{1}}{u_{2}-u_{1}} a_{2}\right) x+\alpha\right]+$

$\lambda_{2}\left[\frac{1}{2} x^{T} B_{\bar{v}} x+\left(\left(b_{1}+\frac{\omega_{1} u_{2}-\omega_{2} u_{1}}{u_{2}-u_{1}}+\bar{v} \frac{\varsigma_{2}-\varsigma_{1}}{v_{2}-v_{1}}\right) b_{2}\right) x+\beta\right] \geq 0$,

$\forall x \in \mathbb{R}^{n}$.

Therefore, there exist $(\bar{u}, \bar{v}, \bar{\omega}, \bar{\varsigma}) \in \mathcal{U}$ and $\left(\lambda_{1}, \lambda_{2}\right) \in \mathbb{R}_{+}^{2} \backslash\{(0,0)\}$ such that relation (13) holds with:

$\bar{\omega}=\frac{\omega_{1} u_{2}-\omega_{2} u_{1}}{u_{2}-u_{1}}+\bar{u} \frac{\omega_{2}-\omega_{1}}{u_{2}-u_{1}}$ and $\bar{\varsigma}=\frac{\varsigma_{1} v_{2}-\varsigma_{2} v_{1}}{v_{2}-v_{1}}+\bar{v} \frac{\varsigma_{2}-\varsigma_{1}}{v_{2}-v_{1}}$

Second case: $u_{1}<u_{2}$ and $v_{1}=v_{2}=\bar{v}$.

Let

$$
\begin{aligned}
& M_{1}=\left(\begin{array}{cc}
A_{1} & a_{1}+\frac{\omega_{1} u_{2}-\omega_{2} u_{1}}{u_{2}-u_{1}} a_{2} \\
a_{1}^{T}+\frac{\omega_{1} u_{2}-\omega_{2} u_{1}}{u_{2}-u_{1}} a_{2}^{T} & 2 \alpha
\end{array}\right), \\
& M_{2}=\left(\begin{array}{cc}
A_{2} & \frac{\omega_{2}-\omega_{1}}{u_{2}-u_{1}} a_{2} \\
\frac{\omega_{2}-\omega_{1}}{u_{2}-u_{1}} a_{2}^{T} & 0
\end{array}\right), M_{3}=\left(\begin{array}{ll}
B_{\bar{v}} & b_{1} \\
b_{1}^{T} & 2 \beta
\end{array}\right) \text { and } \\
& M_{4}=\left(\begin{array}{cc}
0_{\mathbb{S}_{n}(\mathbb{R})} & b_{2} \\
b_{2}{ }^{T} & 0
\end{array}\right) .
\end{aligned}
$$

Then, we have the following linear matrix equalities :

$$
\begin{aligned}
& M_{1}+u_{1} M_{2}=H_{1} ; \quad M_{1}+u_{2} M_{2}=H_{2} ; \\
& M_{3}+\varsigma_{1} M_{4}=H_{3} \text { and } M_{3}+\varsigma_{2} M_{4}=H_{4} .
\end{aligned}
$$

From system $\left(S_{2}\right)$, we obtain that the system

$$
\left\{\begin{array}{l}
\frac{1}{2}\left(\begin{array}{l}
x \\
t
\end{array}\right)^{T}\left(M_{1}+u M_{2}\right)\left(\begin{array}{l}
x \\
t
\end{array}\right)<0, \forall \mathrm{u} \in \mathcal{U}_{1}, \\
\frac{1}{2}\left(\begin{array}{c}
x \\
t
\end{array}\right)^{T}\left(M_{1}+\varsigma M_{2}\right)\left(\begin{array}{l}
x \\
t
\end{array}\right)<0, \forall \varsigma \in \mathcal{U}_{2},
\end{array}\right.
$$

has no solution.

Since the set $\Xi_{\bar{x}}$ is convex, we can apply Theorem 2, thus we can find $\left(\lambda_{1}, \lambda_{2}\right)$ in $\mathbb{R}_{+}^{2} \backslash\{(0,0)\}$ and $(\bar{u}, \bar{\varsigma}) \in \mathcal{U}_{1} \times \mathcal{U}_{4}$ such that

$\lambda_{1}\left[\frac{1}{2}\left(\begin{array}{l}x \\ t\end{array}\right)^{T}\left(M_{1}+\bar{u} M_{2}\right)\left(\begin{array}{l}x \\ t\end{array}\right)\right]+$

$\lambda_{2}\left[\frac{1}{2}\left(\begin{array}{l}x \\ t\end{array}\right)^{T}\left(M_{3}+\bar{\varsigma} M_{4}\right)\left(\begin{array}{l}x \\ t\end{array}\right)\right] \geq 0, \forall(x, t) \in \mathbb{R}^{n} \times \mathbb{R}$. 
Letting $\mathrm{t}=1$, one has:

$\lambda_{1}\left[\frac{1}{2} x^{T} A_{\bar{u}} x+\left(a_{1}+\left(\frac{\omega_{1} u_{2}-\omega_{2} u_{1}}{u_{2}-u_{1}}\right)+\bar{u} \frac{\omega_{2}-\omega_{1}}{u_{2}-u_{1}} a_{2}\right) x+\alpha\right]+$

$\lambda_{2}\left[\frac{1}{2} x^{T} B_{\bar{v}} x+b_{\bar{\varsigma}}^{T} x+\beta\right] \geq 0, \forall x \in \mathbb{R}^{n}$.

Hence, there exist $(\bar{u}, \bar{v}, \bar{\omega}, \bar{\varsigma}) \in \mathcal{U}$ and $\left(\lambda_{1}, \lambda_{2}\right) \in \mathbb{R}_{+}^{2} \backslash\{(0,0)\}$ such that relation (13) holds with :

$$
\bar{\omega}=\frac{\omega_{1} u_{2}-\omega_{2} u_{1}}{u_{2}-u_{1}}+\bar{u} \frac{\omega_{2}-\omega_{1}}{u_{2}-u_{1}}
$$

Third case : $u_{1}=u_{2}=\bar{u}$ and $v_{1}<u_{2}$

Let

$$
\begin{aligned}
M_{1} & =\left(\begin{array}{ll}
A_{\bar{u}} & a_{1} \\
a_{1}^{T} & 2 \alpha
\end{array}\right) ; M_{2}=\left(\begin{array}{cc}
0_{\mathbb{S}_{n}(\mathbb{R})} & a_{2} \\
a_{2}{ }^{T} & 0
\end{array}\right) ; \\
M_{3} & =\left(\begin{array}{cc}
B_{1} & b_{1}+\frac{\omega_{1} u_{2}-\omega_{2} u_{1}}{u_{2}-u_{1}} b_{1} \\
b_{1}^{T}+\frac{\varsigma_{1} v_{2}-\varsigma_{2} v_{1}}{v_{2}-v_{1}} b_{1}{ }^{T} & 2 \beta
\end{array}\right) \text { and } \\
M_{4} & =\left(\begin{array}{cc}
B_{2} & \frac{\varsigma_{2}-\varsigma_{1}}{v_{2}-v_{1}} b_{2} \\
\frac{\varsigma_{2}-\varsigma_{1}}{v_{2}-v_{1}} b_{2}{ }^{T} & 0
\end{array}\right) .
\end{aligned}
$$

We proceed like in the second case by changing the role of $\mathrm{u}$ and $\mathrm{v}$ with

$$
\bar{\zeta}=\frac{\varsigma_{1} v_{2}-\varsigma_{2} v_{1}}{v_{2}-v_{1}}+\bar{v} \frac{\varsigma_{2}-\varsigma_{1}}{v_{2}-v_{1}} \text {. }
$$

Fourth case: $u_{1}=u_{2}=\bar{u}$ and $v_{1}=v_{2}=\bar{v}$.

Putting :

$$
M_{1}=\left(\begin{array}{cc}
A_{\bar{u}} & a_{1} \\
a_{1}^{T} & 2 \alpha
\end{array}\right) ; M_{2}=\left(\begin{array}{cc}
0_{\mathbb{S}_{n}(\mathbb{R})} & a_{2} \\
a_{2}^{T} & 0
\end{array}\right) ; M_{3}=\left(\begin{array}{cc}
B_{\bar{v}} & b_{1} \\
b_{1}^{T} & 2 \beta
\end{array}\right) \text { and } \quad M_{4}=\left(\begin{array}{cc}
0_{\mathbb{S}_{n}(\mathbb{R})} & b_{2} \\
b_{2}^{T} & 0
\end{array}\right) .
$$

In this case, we remark that :

$$
\begin{aligned}
& M_{1}+\omega_{1} M_{2}=H_{1} ; \quad M_{1}+\omega_{2} M_{2}=H_{2} ; \\
& M_{3}+\varsigma_{1} M_{4}=H_{3} \text { and } M_{3}+\varsigma_{2} M_{4}=H_{4} .
\end{aligned}
$$

The system

$$
\left\{\begin{array}{l}
\frac{1}{2}\left(\begin{array}{l}
x \\
t
\end{array}\right)^{T}\left(M_{1}+\omega M_{2}\right)\left(\begin{array}{l}
x \\
t
\end{array}\right)<0, \forall \omega \in \mathcal{U}_{3}, \\
\frac{1}{2}\left(\begin{array}{l}
x \\
t
\end{array}\right)^{T}\left(M_{1}+\varsigma M_{2}\right)\left(\begin{array}{l}
x \\
t
\end{array}\right)<0, \forall \varsigma \in \mathcal{U}_{2},
\end{array}\right.
$$

has no solution because it is equivalent to another one like $\left(S_{2}\right)$. 
Again from the convexity of $\Xi_{\bar{x}}$ and Theorem 2, there exist

$\left(\lambda_{1}, \lambda_{2}\right) \in \mathbb{R}_{+}^{2} \backslash\{(0,0)\}$ and $(\bar{\omega}, \bar{\varsigma}) \in \mathcal{U}_{3} \times \mathcal{U}_{4}$ such that

$\lambda_{1}\left[\frac{1}{2}\left(\begin{array}{l}x \\ t\end{array}\right)^{T}\left(M_{1}+\bar{\omega} M_{2}\right)\left(\begin{array}{l}x \\ t\end{array}\right)\right]+$

$\lambda_{2}\left[\frac{1}{2}\left(\begin{array}{c}x \\ t\end{array}\right)^{T}\left(M_{3}+\bar{\varsigma} M_{4}\right)\left(\begin{array}{c}x \\ t\end{array}\right)\right] \geq 0, \forall(x, t) \in \mathbb{R}^{n} \times \mathbb{R}$.

Letting $\mathrm{t}=1$, one has:

$\lambda_{1}\left[\frac{1}{2} x^{T} A_{\bar{u}} x+a_{\bar{\omega}}^{T} x+\alpha\right]+$

$\lambda_{2}\left[\frac{1}{2} x^{T} B_{\bar{v}} x+b_{\bar{\varsigma}}^{T} x+\beta\right] \geq 0, \forall x \in \mathbb{R}^{n}$.

Finally, in all cases, there exist $(\bar{u}, \bar{v}, \bar{\omega}, \bar{\zeta}) \in \mathcal{U}$ and $\left(\lambda_{1}, \lambda_{2}\right) \in \mathbb{R}_{+}^{2} \backslash\{(0,0)\}$ such that (13) holds.

From Slater condition, one obtains that: $\lambda_{1}>0$ and

$\left(\frac{1}{2} x^{T} A_{\bar{u}} x+a_{\bar{\omega}}^{T} x+\alpha\right)+\lambda\left(\frac{1}{2} x^{T} B_{\bar{v}} x+b_{\bar{\zeta}}^{T} x+\beta\right) \quad \geq 0, \forall x \in \mathbb{R}^{n},(14)$

with: $\lambda=\frac{\lambda_{2}}{\lambda_{1}}$.

From this inequality (14), we get:

$$
\max _{(\boldsymbol{u}, \boldsymbol{\omega}) \in \mathcal{U}_{1} \times \mathcal{U}_{3}}\left(\frac{1}{2} x^{T} A_{u} \mathrm{x}+a_{\omega}^{T} x\right)+\alpha+\lambda\left(\frac{1}{2} x^{T} B_{\bar{v} \mathrm{x}}+b_{\bar{\varsigma}}^{T} x+\beta\right) \geq 0, \quad \forall x \in \mathbb{R}^{n} .
$$

For $x=\bar{x}$, since $\bar{x}$ is a feasible solution of (NH-QCQP), we obtain:

$$
\left(\frac{1}{2} \bar{x}^{T} B_{\bar{v}} \bar{x}+b_{\bar{\zeta}}^{T} \bar{x}+\beta\right)=0 \text {. }
$$

Let us consider:

$\mathbb{R}^{n} \rightarrow \mathbb{R}, \mathrm{x} \mapsto \phi(\mathrm{x}):=\frac{1}{2} x^{T} A_{\bar{u}} \mathrm{x}+a_{\bar{\omega}}^{T} x+\alpha+\lambda\left(\frac{1}{2} x^{T} B_{\bar{v}} \mathrm{x}+b_{\bar{\varsigma}}^{T} x+\beta\right)$

By using the definition of $\alpha$, (14) and (15), we get:

$\phi(\mathrm{x}) \geq 0$ and $\phi(\bar{x}) \leq 0$. It is follows that $\phi(\mathrm{x}) \geq \phi(\bar{x}) . \bar{x}$ minimize $\phi$ on $\mathbb{R}^{n}$ and $\varphi$ is twice differentiable. Then, $\nabla \phi(\bar{x})=0$ and $\nabla^{2} \phi(\bar{x}) \geqslant 0$.

Therefore,

$$
\left\{\begin{array}{l}
\left(A_{\bar{u}}+\lambda B_{\bar{v}}\right) \bar{x}+a_{\bar{\omega}}^{T}+b_{\bar{\varsigma}}^{T}=0 \\
A_{\bar{u}}+\lambda B_{\bar{v}} \geqslant 0 .
\end{array}\right.
$$

[(ii) $=)(\mathrm{i})]$. Suppose there exist $\lambda \geq 0$ and $(\bar{u}, \bar{v}, \bar{\omega}, \bar{\varsigma}) \in \mathcal{U}$

$$
\begin{cases}\left(A_{\bar{u}}+\lambda B_{\bar{v}}\right) \bar{x}+a_{\bar{\omega}}+\lambda b_{\bar{\varsigma}}=0, & \text { (First-order Condition) } \\ \lambda\left(\frac{1}{2} \bar{x}^{T} B_{\bar{v}} \bar{x}+b_{\bar{\varsigma}}^{T} \bar{x}+\beta\right)=0, & \text { (Complementary Slackness) } \\ A_{\bar{u}}+\lambda B_{\bar{v}} \succcurlyeq 0 . & \text { (Second-order Condition) }\end{cases}
$$


Let us consider the map,

$\psi: \mathbb{R}^{n} \rightarrow \mathbb{R}, \mathrm{x} \longmapsto \psi(\mathrm{x}):=\frac{1}{2} x^{T} A_{\bar{u}} \mathrm{x}+a_{\bar{\omega}}^{T} x+\lambda\left(\frac{1}{2} x^{T} B_{\bar{v}} \mathrm{x}+b_{\bar{\zeta}}^{T} x+\beta\right)$

and assume that $x$ be a robust feasible solution of (NH-QCQP).

First-order condition and second-order condition gives us that

$\nabla \psi(\bar{x})=0$ and $\psi$ is convex. Then, $\bar{x}$ is a global minimizer of $\psi$ and one has

$$
\begin{aligned}
\psi(\mathrm{x}) \geq \psi(\bar{x}) \Longrightarrow & \frac{1}{2} x^{T} A_{\bar{u}} \mathrm{x}+a_{\bar{\omega}}^{T} x \geq \frac{1}{2} \bar{x}^{T} A_{\bar{u}} \bar{x}+a_{\bar{\omega}}^{T} \bar{x} \\
& +\lambda\left(\frac{1}{2} \bar{x}^{T} B_{\bar{v}} \bar{x}+b_{\bar{\varsigma}}^{T} \bar{x}+\beta\right)-\lambda\left(\frac{1}{2} x^{T} B_{\bar{v}} \mathrm{x}+b_{\bar{\varsigma}}^{T} x+\beta\right) \\
\Rightarrow & \frac{1}{2} x^{T} A_{\bar{u}} \mathrm{x}+a_{\bar{\omega}}^{T} x \geq \frac{1}{2} \bar{x}^{T} A_{\bar{u}} \bar{x}+a_{\omega}^{T} \bar{x} \\
& \quad-\lambda\left(\frac{1}{2} x^{T} B_{\bar{v} \mathrm{x}}+b_{\bar{\varsigma}} \mathrm{x}+\beta\right) \\
\Rightarrow & \frac{1}{2} x^{T} A_{\bar{u}} \mathrm{x}+a_{\bar{\omega}}^{T} x \geq \frac{1}{2} \bar{x}^{T} A_{\bar{u}} \bar{x}+a_{\omega}^{T} \bar{x} .
\end{aligned}
$$

Therefore, $\bar{x}$ is robust optimal solution of (NH-QCQP).

\section{Optimistic Dual and Strong Robust Duality of QCQOP}

In this section, we characterize the solutions of dual optimistic. We also show that the robust strong duality property holds.

Let us consider

$\mathbb{R}^{n} \times \mathcal{U} \times \mathbb{R}^{+} \rightarrow \mathbb{R},(\mathrm{x}, \xi, \lambda) \mapsto \mathcal{L}(\mathrm{x}, \xi, \lambda):=q_{0}(x, \xi)+\lambda q_{1}(x, \xi)$

where $\xi=(u, v, \omega, \varsigma), \quad q_{0}(x, \xi):=\frac{1}{2} x^{T} A_{u} \mathrm{x}+a_{\omega}^{T} x$

and $q_{1}(x, \xi):=\frac{1}{2} x^{T} B_{v} \mathrm{x}+b_{\varsigma}^{T} x+\beta$.

The problem

$$
\begin{aligned}
& \max \inf _{x \in \mathbb{R}^{n}} \mathcal{L}(\mathrm{x}, \xi, \lambda) \quad \text { (OD-QCQP) } \\
& \text { s.t. }(\xi, \lambda) \in \mathcal{U} \times \mathbb{R}^{+}
\end{aligned}
$$

is called the optimistic dual of (NH-QCQP).

Theorem 5 Assume that assumptions of Theorem 4 hold. If $\bar{x}$ is a robust solution of (NH-QCQP), then there exist $(\bar{\xi}, \bar{\lambda}) \in \mathcal{U} \times \mathbb{R}^{+}$such that $(\bar{\xi}, \bar{\lambda})$ is an optimal solution of (OD-QCQP).

Proof. From Theorem 4, there exists $(\bar{u}, \bar{v}, \bar{\omega}, \bar{\zeta}, \bar{\lambda}) \in \mathcal{U} \times \mathbb{R}^{+}$such that

$$
\begin{cases}\left(A_{\bar{u}}+\lambda B_{\bar{v}}\right) \bar{x}+a_{\bar{\omega}}+\lambda b_{\bar{\varsigma}}=0, & \text { (First-order Condition) } . \\ \lambda\left(\frac{1}{2} \bar{x}^{T} B_{\bar{v}} \bar{x}+b_{\bar{\varsigma}}+\beta\right)=0, & \text { (Complementary Slackness) } \\ A_{\bar{u}}+\lambda B_{\bar{v}} \geqslant 0 . & \text { (Second-order Condition) }\end{cases}
$$


We set $\bar{\xi}=(\bar{u}, \bar{v}, \bar{\omega}, \bar{\zeta})$. For all feasible solution

$(u, v, \omega, \varsigma, \lambda) \in \mathcal{U} \times \mathbb{R}^{+}$of (OD-QCQP), one has :

$$
\begin{aligned}
\mathcal{L}(\bar{x}, \bar{\xi}, \bar{\lambda}) & =q_{0}(\bar{x}, \bar{\xi})+\bar{\lambda} q_{1}(\bar{x}, \bar{\xi}) \\
& =q_{0}(\bar{x}, \bar{\xi}) \\
& =\max _{\xi \in \mathcal{U}} q_{0}(\bar{x}, \xi) \\
& \geq q_{0}(\bar{x}, \xi), \forall \xi \in U \\
& \geq q_{0}(\bar{x}, \xi)+\lambda q_{1}(\bar{x}, \xi), \forall(\xi, \lambda) \in U \times \mathbb{R}^{+} \\
& =\mathcal{L}(\bar{x}, \bar{\xi}, \lambda), \forall(\xi, \lambda) \in \mathcal{U} \times \mathbb{R}^{+} .
\end{aligned}
$$

Hence, $(\bar{\xi}, \bar{\lambda})$ is an optimal solution of (OD-QCQP).

Corollary 2 Suppose that assumptions of Theorem 5 hold.

If (RNH-QCQP) has an optimal solution, then the strong duality property is fulfilled.

Proof. Let $\bar{x}$ be an optimal solution of (RNH-QCQP). So,

Moreover,

$$
\begin{aligned}
\min (\mathrm{RNH}-\mathrm{QCQP}) & =\frac{1}{2} \max _{u \in \mathcal{U}_{1}} \bar{x}^{T} A_{u} \bar{x}+\max _{\omega \in \mathcal{U}_{3}} a_{\omega}^{T} \bar{x} \\
& =\frac{1}{2} \bar{x}^{T} A_{u} \bar{x}+a_{\omega}^{T} \bar{x} .
\end{aligned}
$$

$$
\begin{aligned}
\max (\mathrm{OD}-\mathrm{QCQP}) & =\max _{(\xi, \lambda) \in \mathcal{U} \times \mathbb{R}^{+}} \inf _{x \in \mathbb{R}^{n}} \mathcal{L}(\mathrm{x}, \xi, \lambda) \\
& =\mathcal{L}(\bar{x}, \bar{\xi}, \bar{\lambda}) \\
= & q_{0}(\bar{x}, \bar{\xi})+\bar{\lambda} q_{1}(\bar{x}, \bar{\xi}) \\
= & \frac{1}{2} \bar{x}^{T} A_{u} \bar{x}+a_{\omega}^{T} \bar{x} .
\end{aligned}
$$

Thus, $\min (\mathrm{RNH}-\mathrm{QCQP})=\max (\mathrm{OD}-\mathrm{QCQP})$.

\section{Example}

We deal with the following 2-dimensions QCQOP

where:

$$
\begin{aligned}
& \min _{x \in \mathbb{R}^{n}} \frac{1}{2} x^{T} A_{u} \mathrm{x}, \\
& \text { s.t. } \frac{1}{2} x^{T} B_{v} \mathrm{x}+\beta \leq 0,
\end{aligned}
$$

$$
\begin{aligned}
& A_{0}=\left(\begin{array}{cc}
\frac{1}{2} & -\frac{3}{2} \\
-\frac{3}{2} & \frac{1}{2}
\end{array}\right), A_{1}=\left(\begin{array}{ll}
1 & 0 \\
0 & 1
\end{array}\right), B_{0}=\left(\begin{array}{cc}
1 & \frac{1}{2} \\
\frac{1}{2} & 1
\end{array}\right) \\
& B_{1}=\left(\begin{array}{cc}
-5 & -10 \\
-10 & -5
\end{array}\right), \beta=-1,|u| \leq 10^{-1} \text { and }|v| \leq 5 \cdot 10^{-1} .
\end{aligned}
$$


With the interval interpolation approach regarding to :

-matrices $A_{0}$ and $A_{1}, A_{u}=A_{0}+u A_{1}=\left(\begin{array}{cc}\frac{2 u+1}{2} & -\frac{3}{2} \\ -\frac{3}{2} & \frac{2 u+11}{2}\end{array}\right)$,

-matrices $B_{0}$ and $B_{1}, B_{v}=B_{0}+v B_{1}=\left(\begin{array}{cc}1-5 v & \frac{1-20 v}{2} \\ \frac{1-20 v}{2} & 1-5 v\end{array}\right)$.

Let $x_{0}=\left(\frac{1}{2}, \frac{1}{2}\right)^{T}$.Then we verify that the strictly feasibility condition holds with this point $x_{0}$. The matrices $A_{u_{1}}, A_{u_{2}}, B_{v_{1}}$ and $B_{v_{2}}$ commute. From Proposition 1 , the set $\Xi=\left\{\left(x^{T} A_{u_{1}} x, x^{T} A_{u_{2}} x, x^{T} B_{v_{1}} x, x^{T} B_{v_{2}} x\right) \mid x \in \mathbb{R}^{n}\right\}$

is convex. Where:

$A_{u_{1}}=\left(\begin{array}{cc}\frac{2}{5} & -\frac{3}{2} \\ -\frac{3}{2} & \frac{2}{5}\end{array}\right), A_{u_{2}}=\left(\begin{array}{cc}\frac{3}{5} & -\frac{3}{2} \\ -\frac{3}{2} & \frac{3}{5}\end{array}\right), B_{v_{1}}=\left(\begin{array}{cc}\frac{5}{4} & 1 \\ 1 & \frac{5}{4}\end{array}\right)$ and $B_{v_{2}}=\left(\begin{array}{cc}\frac{3}{4} & 0 \\ 0 & \frac{3}{4}\end{array}\right)$.

The problem $(\mathcal{P})$ can be rewritten under the analytically form still denoted $(\mathcal{P})$ and given by:

$$
\begin{aligned}
& \min _{x \in \mathbb{R}^{n}} \frac{1}{4}(2 u+1) x_{1}^{2}-\frac{3}{2} x_{1} x_{2}+\frac{1}{4}(2 u+1) x_{2}^{2}, \\
& \text { s.t. } \frac{1}{2}(1-5 v) x_{1}^{2}+\frac{1}{2}(1-20 v) x_{1} x_{2}+\frac{1}{2}(1-5 v) x_{2}^{2} \leq 0 .
\end{aligned}
$$

From Theorem 3, we deduce that the robust solution of $(\mathcal{P})$ satisfies

$$
\begin{cases}{[2 u+1+2 \lambda(1-5 v)] x_{1}+[-3+\lambda(1-20 v)] x_{2}} & =0 \\
{[-3+\lambda(1-20 v)] x_{1}+[2 u+1+2 \lambda(1-5 v)] x_{2}} & =0 \\
\lambda\left[\frac{1}{2}(1-5 v) x_{1}^{2}+\frac{1}{2}(1-20 v) x_{1} x_{2}+\frac{1}{2}(1-5 v) x_{2}^{2}-1\right]=0 \\
\left(\begin{array}{cc}
\frac{2 u+1}{2}+\lambda(1-5 v) & \frac{1}{2}(-3+\lambda(1-20 v)) \\
\frac{1}{2}(-3+\lambda(1-20 v)) & \frac{2 u+1}{2}+\lambda(1-5 v)
\end{array}\right) & \geqslant 0\end{cases}
$$

Letting $\bar{u}=3.10^{-2}, \bar{v}=4.10^{-2}$ and $\bar{\lambda}=\frac{97}{90}$. The resolution of this system gives us the following robust solutions:

$$
\bar{x}= \pm\left(\frac{\sqrt{10}}{3}, \frac{\sqrt{10}}{3}\right)^{T}
$$




\section{Conclusion}

We have dealt with a non-convex quadratic optimization problem subjected to data uncertainties both in constraint and in criterion. Such an optimization model is extremely difficult to solve thanks its two levels of optimization (in short "mini-max"). We propose a new definition of convexity for a RO problem and an adequate $\mathrm{S}$-procedure in order to characterize the global robust optimal solution of these quadratic uncertain problem in homogeneous case in one hand. We have also dealt with the non-homogeneous case in other hand. The example given in Section 6 allows to validate this paradigm in the reality. In this work, we bring some necessary and sufficient conditions in Theorem 5 and Corollary 2 to make strong duality property holds. A next step could be to address some numerical optimization algorithms for solving a large scale of these kind of QCQOPs.

\section{References}

[1] Moussa, B.(2016). Contribution à l'étude de la robustesse et à la dualité en optimisation, Thèse, Université d'Avignon, France.

[2] Ben-Tal, A., El Ghaoui A. L. and Nemirovski, A. (2009). Robust Optimization, Princeton Series in Applied Mathematics, Princeton University Press.

[3] Ben-Tal, A. and Nemirovski, A. (1998). Robust Convex Optimization, Mathematics of Operations Research, 23(4), 769-805.

[4] Ben-Tal, A. and Nemirovski, A. (1999). Robust solutions of uncertain linear programs, Operations Research Letters, Elsevier B.V., 25(1), 1-13.

[5] Ben-Tal, A. and Nemirovski, A. (2012). Lectures on modern convex optimization: analysis, algorithms and engineering applications, MPS-SIAM Series on Optimization, Society for Industrial and Applied Mathematics.

[6] Bertsimas, D. and Sim, M. (2003). Robust discrete optimization and network flows, Mathematical Programming, Springer-Verlag, 98(1), 49-71.

[7] Bertsimas, D. and Sim, M. (2004). The Price of Robustness, Operations Research, INFORMS, (52) $1,35-53$.

[8] Bertsimas, D. and Sim, M. (2005). Tractable Approximations to Robust Conic Optimization Problems, Mathematical Programming, Springer-Verlag, 107(1), 5-36.

[9] Birge, J. R. and Louveaux, F. (1997). Introduction to Stochastic Programming, SpringerVerlag, New York, NY, USA.

[10] Bonnans, J., Gilbert J. C, Lemaréchal, C. and C. Sagastizábal.(2006) Numerical Optimization : Theoretical and Practical Aspects, Second Edition, Springer-Verlag Berlin Heidelberg, NewYork.

[11] Boyd, S. and Vandenberghe, L. (2004). Convex Optimization, Cambridge University Press.

[12] Brezis, H. (1983). Analyse fonctionnelle : Théorie et Applications, Collection Mathématiques pour la maitrise, MASSON, Paris.

[13] Brickman, L. (1961). On the field of values of a matrix, Proceedings of the American Mathematical Society, 12, 61-66.

[14] Conn, A., Scheinberg K. and Vicente, L. (2009). Introduction to Derivative-Free Optimization, MPS-SIAM Series on Optimization.

[15] Dantzig, G. B. (1955). Linear Programming under Uncertainty, Management Science, 1(3\&4), 197-206.

[16] Dines, L. L. (1941). On the mapping of quadratic forms, Bulletin of the American Mathematical Society, 47(6), 494-498. 
[17] Ghaoui L. El. and Lebret, H.(1997). Robust Solutions to Least-Squares Problems with Uncertain Data, SIAM Journal on Matrix Analysis and Applications, Society for Industrial and Applied Mathematics, 18(4), 1035-1064.

[18] Ghaoui, L. El., Oustry, F. and Lebret H. (1998). Robust Solutions to Uncertain Semidefinite Programs, SIAM Journal on Optimization, 9(1), 33-52.

[19] Eva, B.-F., David L. and Andrew R. (July 5-9, 1999). Quadratic Forms and Their Applications, Proceedings of the Conference on Quadratic Forms and Their Applications, , University College Dublin, Contemporary mathematics, Vol. 272, American Mathematical Society, 2000.

[20] Fletcher, R. (1987). Practical Methods of Optimization, Second Edition, John Wiley \& Sons, NewYork.

[21] Gould, F. J. (1971). Proximate Linear Programming : AnExperimental Study of a Modified Simplex Algorithm for Solving Linear Programs with Inexact Data, Statistics Mimeo Series (789). Department of Statistics, University of North Carolina, Chapel Hill, North Carolina.

[22] Gupta, S. K. and Rosenhead, J. (1968). Robustness in sequential investment decisions, Management Science, 15(2), 18-29.

[23] Hiriart Urruty, J.-B. (2001). Global optimality conditions in maximizing a convex quadratic function under convex quadratic constraints, Journal of Global Optimization, 21, 445-455.

[24] Hiriart Urruty, J.-B. and Lemaréchal, C. (1993). Convex Analysis and Minimization Algorithms I : Fundamentals, Grundlehren der mathematischen Wissenschaften, SpringerVerlag, Berlin, New York, 305.

[25] Hiriart Urruty, J.-B. and Lemaréchal C. (1993). Convex Analysis and Minimization Algorithms II : Advanced Theory and Bundle Methods, Grundlehren der mathematischen Wissenschaften, 306, Springer-Verlag, Berlin, New York.

[26] Houska, B. (2011). Robust Optimization of Dynamic Systems,PhD Thesis.

[27] Infange, G. (2011). Stochastic Programming, The State of the Art In Honor of George B. Dantzig, Springer New York Dordrecht Heidelberg London.

[28] Jeyakumar, V., Huy, N. Q. and Li, G. (2009). Necessary and sufficient conditions for Slemma and nonconvex quadratic optimization, Optim. Eng, 10, 491-503.

[29] Jeyakumar, V., Lee G. M. and Li. G. Y. (2009). Alternative theorems for quadratic inequality systems and global quadratic optimization, SIAM J. Optim, 2, 983-1001.

[30] Jeyakumar, V. and Li, G. (2010). Strong duality in robust convex programming : complete characterizations, SIAM J. Optim.,3384-3407.

[31] Jeyakumar, V. and Li, G. Y. (2012). Robust solutions of quadratic optimization over single quadratic constraint under interval uncertainty, J. Glob. Optim.

[32] Kall, P. and Wallace, S. (1994). Stochastic Programming, John Wiley \& Sons, New York, Chichester.

[33] Khargonekar, P. P., Petersen I. R. and Zhou, K. (1990). Robust stabilization of uncertain linear systems: quadratic stabilizability and H/sup infinity / control theory, IEEE Transactions on Automatic Control, 35(3), 356-361.

[34] Kouvelis, P. and Yu, G. (1997). Robust Discrete Optimization and Its Applications, Nonconvex Optimization and Its Applications, Kluwer Academic Publishers \& Springer Science+Business Media Dordrecht - Verlag US, 14.

[35] Li, G., Jeyakumar V. and Lee, G. M. (2011). Robust conjugate duality for convex optimization under uncertainty with application to data classification, Nonlinear Analysis, 74, 2327-2341. 
[36] Li, G., Wu Z. and Quan, J. (2015). Global optimality for nonconvex minimization problems with quadratic constraints, Journal of inequalities and applications, 257.

[37] Marti, K. (2008). Stochastic Optimization Methods, Second Edition, Springer-Verlag Berlin Heidelberg.

[38] Mulvey, J., Vanderbei, R. and Zenios, S. (1995). Robust Optimization of Large-Scale Systems, Operations Research, INFORMS, 43(2), 264-281.

[39] Nesterov, Y. (2014). Introductory Lectures on Convex Optimization : A Basic Course. Springer publishing Company, Incorporated Optimization, 1 edition.

[40] Nocedal, J. and Wright, S. (2006). Numerical Optimization, Second Edition, Springer Science+Business Media, LLC.

[41] Olik, I. and Terlaky, T. (2007). A survey of the S-lemma, SIAM Rev. 49, 371-418.

[42] Polyak, B. (1998). Convexity of Quadratic Transformations and Its Use in Control and Optimization, Journal of Optimization Theory and Applications, 99(3), 553-583.

[43] Rockafellar, R. T. and Wets, R. J.-B. (1998). Variational Analysis, Grundlehren der mathematischen Wissenschaften, Springer-Verlag Berlin Heidelberg London New York, (317), Corrected 3rd printing 2009.

[44] Rosenhead, J., Elton, M. C. J. and Gupta, S. K. (Dec., 1972). Robustness and Optimality as Criteria for Strategic Decisions, Operational Research Quarterly (1970-1977), 23 (4), 413431.

[45] Sanogo, S. (Février 2016). Conception Optimale de Circuits Magnétiques dédiés à la Propulsion Spatiale Electrique par des Méthodes d'Optimisation Topologique, Thèse, LAPLACE, UPS-Toulouse, France.

[46] Shapiro, A., Dentcheva, D. and Ruszczyński, A. (2009). Lectures on Stochastic Programming: Modeling and Theory, Society for Industrial and Applied Mathematics and the Mathematical Programming Society.

[47] Shapiro, A. and Ruszczyński, A. (2003). Stochastic Programming, Operations Research and Management Science, Science Direct,Elsevier B.V.

[48] Sim, M. (2004). Robust Optimization, PhD Thesis, Operation Research, Massachusetts Institute of Technology.

[49] Soyster, A. L. (1973). Convex programming with set-inclusive constraints and applications to inexact linear programming, Operations Research, 21(5), 1154-1157. 